\title{
Annex 3
}

\section{Deployment Plan}

\section{Lesotho 2012 Parliamentary Elections}

\begin{tabular}{|l|l|}
\hline TEAM/DISTRICTS & OBSERVERS \\
\hline 1. Maseru/Berea/Mafeteng & $\begin{array}{l}\text { H.E. Dr Bakili Muluzi (Chair) } \\
\text { Mr Linford Andrews } \\
\text { Mr Julius Mucunguzi } \\
\text { Mrs Madonna Lynch }\end{array}$ \\
\hline 2. Qacha's Nek & Mr Aloisious Nthenda \\
\hline 3. Quthing / Mohale's Hoek & $\begin{array}{l}\text { Mr Orin Gordon } \\
\text { Dr Tres-Ann Kremer }\end{array}$ \\
\hline 4. Leribe / Butha-Buthe & $\begin{array}{l}\text { Mrs Rina Ray } \\
\text { Mr Med S K Kaggwa }\end{array}$ \\
\hline 5. Thaba-Tseka & $\begin{array}{l}\text { Dr Rajendra Prasad } \\
\text { Ms Aminath Majdha }\end{array}$ \\
\hline
\end{tabular}

